\title{
FRACTIONALLY SPACED CMA EQUALIZERS UNDER PERIODIC AND CORRELATED INPUTS
}

\author{
James P. LeBlanc ${ }^{1} \quad$ Inbar Fijalkow ${ }^{2} \quad$ Birkett Huber ${ }^{3} \quad$ C. Richard Johnson, Jr. ${ }^{1}$ \\ ${ }^{1}$ School of Elec. Eng. \\ Cornell University \\ ${ }^{2}$ ENSEA, Groupe ETIS \\ 95014 Cergy-Pontoise Cedex, France \\ ${ }^{3}$ Math Dept. \\ (GdR Signal et Image) \\ Cornell University \\ Ithaca, NY USA
}

\begin{abstract}
CMA Fractionally Spaced Equalizers (CMA-FSEs) have been shown, under certain conditions, to be globally asymptotically convergent to a setting which provides perfect equalization. Such a result relies heavily on the assumptions of a white source and no channel noise (as is the case in much of the literature's analysis of CMA). Herein, we relax the white source assumption and examine the effect of source correlation on CMA. Analytic results are meshed with examples showing CMA-FSE source correlation effects. Techniques for finding all stationary and saddle points on the CMA-FSE error surface are presented using recent developments in the Algebraic-Geometry community.
\end{abstract}

\section{INTRODUCTION}

"There are some aspects of blind deconvolution that do not fit easily into the theory presented in this chapter. The most important one is probably what happens if the input sequence is not white. Little work has been done on this matter." - Bellini [4]

In the equalization problem for digital communication, a source sequence $s(k)$ drawn from alphabet members $\mathcal{A}=$ $\left\{a_{1} \ldots a_{m}\right\}$ is transmitted at a baud rate of $\frac{1}{T}$ through an analog channel $c(t)$ (considered to include any pulse shaping) which introduces intersymbol interference (ISI) on the received signal $r(t)$ which is then sampled at or above the baud rate. The case where the sample period equals $T$ is known as "baud spaced", whereas the case where the sample period is $T / L$ (for $L$ positive integer), is known as "fractionally spaced".

A discrete-time adaptive linear equalizer with tap weight vector $D(k)=\left[\begin{array}{lllll}d_{0}(k) & d_{1}(k) & \ldots & d_{Q}(k)\end{array}\right]$ is then used to reduce the ISI, producing the output signal $y(k)$ as in Figure 1. Proper equalization will result in $y(k)=s(k-\nu)$ with $\nu$ being the channel-equalizer delay. The term blind refers to performing equalizer adaptation without the use of a training signal (à la LMS).

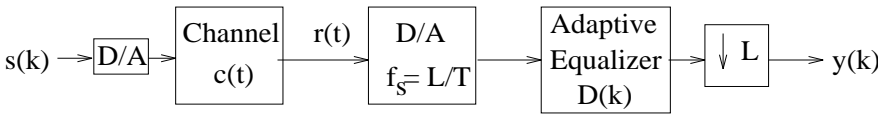

Figure 1: CMA-FSE Block Diagram

In CMA, with a strictly real alphabet, the equalizer taps are updated at baud rate based on the stochastic gradient descent of the non-linear cost function,

$$
J(y)=\frac{1}{4} \mathrm{E}\left\{\left(y^{2}-\gamma\right)^{2}\right\}
$$

\footnotetext{
${ }^{1}$ Supported by Applied Signal Technology, Inc.

${ }^{2}$ Supported by the French Ministère des Affaires Etrangères (bourse Lavoisier) for a postdoctoral visit at Cornell University.
}

where $\gamma=\frac{\mathrm{E}\left\{a^{4}\right\}}{\mathrm{E}\left\{a^{2}\right\}}$, for $a \in \mathcal{A}$. The CMA tap adaptation becomes,

$$
D(k+1)=D(k)-\mu\left(y^{3}(k)-\gamma y(k)\right) X(k)
$$

where the equalizer output is $y(k)=X^{\mathrm{t}}(k) D(k), D(k)$ is the $Q+1 \times 1$ tap vector, $X(k)$ is the equalizer's regressor at time $k$ (i.e. $X(k)=\left[\begin{array}{llll}r(k T) & r\left(k T-\frac{T}{L}\right) & \ldots & r\left(k T-Q \frac{T}{L}\right)\end{array}\right]^{t}$, a $Q+1 \times 1$ vector) and $\mu$ is a small step-size.

In [3] it is noted that the fractionally spaced equalizer problem can be phrased as a multi-channel baud-spaced equalization problem as in Figure 2, shown for the case $L=2$. For a channel $c(t)$ with time support between $P+1$ and $P+2$ baud, each of the $i$ discrete subchannels of length $\frac{P+1}{L}$ is denoted $C_{i}$ and the corresponding sub-equalizer (of length $\left.\frac{Q+1}{L}\right)$ at time $k$ is denoted $D_{i}(k)$. The global system (i.e. the combined channel-equalizer) at time $k$ is then defined by the length $N+1\left(=\frac{P+1}{L}+\frac{Q+1}{L}-1\right)$ column vector $H(k)$ given by

$$
H(k)=\mathbb{C} D(k)
$$

Where $\mathbb{C}$ is the multi-channel convolution matrix as in (4).

$$
\mathbb{C}=\left[\begin{array}{cccccc}
c_{1}(0) & c_{1}(T) & \ldots & c_{1}(P T) & & \\
\vdots & \vdots & \ddots & \vdots & & \\
c_{L}(0) & c_{L}(T) & \ldots & c_{L}(P T) & & \\
& \ddots & \ddots & \ddots & \ddots & \\
& & c_{1}(0) & c_{1}(T) & \ldots & c_{1}(P T) \\
& & \vdots & \vdots & \ddots & \vdots \\
& & c_{L}(0) & c_{L}(T) & \ldots & c_{L}(P T)
\end{array}\right]
$$

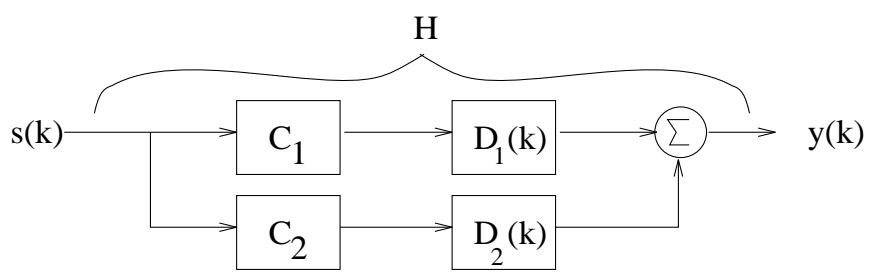

Figure 2: Multichannel Block Diagram

It is shown in [3] that for the CMA-FSE meeting the following conditions:

- Equalizer Length: equalizer time span meets or exceeds the channel time span.

- Sub-Channel Disparity: there exist two sub-channels that have no common roots.

- Noiseless Case: no channel noise is present.

- Source Whiteness: the source is a uniformly distributed iid sequence. 
then the CMA error surface exhibits only global minima with perfect equalizing performance. We now investigate CMAFSE's behavior meeting the length, no noise, and channel diversity conditions, but relax the white source condition to allow for temporally correlated sources.

\section{CMA-FSE STATIONARY POINTS}

The convergent points of the CMA-FSE adaptive equalizer are the minima of the error surface formed by (1). These minima are a subset of the error surface stationary points which are described by $\frac{\partial J}{\partial D}=\overrightarrow{0}$. Consider the equalizer output $y(k)$ due to a source vector $S(k)=\left[\begin{array}{lll}s(k) & \ldots & s(k-N)\end{array}\right]^{t}$. With $y(k)=D^{\mathrm{t}} \mathbb{C}^{\mathrm{t}} S(k)$, the cost function gradient is given by,

$$
\frac{\partial J}{\partial D}=\mathrm{E}\left\{\left(\left(D^{\mathrm{t}} \mathbb{C}^{\mathrm{t}} S\right)^{3}-\gamma D^{\mathrm{t}} \mathbb{C}^{\mathrm{t}} S\right) \mathbb{C} S\right\}
$$

With $H=\mathbb{C} D$, and recognizing $\mathbb{C}$ as a non-random quantity this becomes,

$$
\frac{\partial J}{\partial D}=\mathbb{C E}\left\{\left(\left(H^{\mathrm{t}} S\right)^{3}-\gamma H^{\mathrm{t}} S\right) S\right\}
$$

Since $\mathbb{C}$ is full column rank by channel disparity condition [5], then $\frac{\partial J}{\partial D}=\overrightarrow{0}$ necessarily implies

$$
\mathrm{E}\left\{\left(\left(H^{\mathrm{t}} S\right)^{3}-\gamma H^{\mathrm{t}} S\right) S\right\}=0
$$

Hence, the stationary points of the CMA-FSE equalizer correspond to stationary parameterizations of the global system which are affected only by the source statistics.

The importance of this result is that it allows an analysis of CMA stationary points (and hence equalization behavior) that is independent of a particular channel (meeting the perfect equalization conditions). Furthermore, it allows assessment of correlation effects in terms of the source correlation (as opposed to the received signal correlation). Expanding 7 yields,

$\mathrm{E}\left\{\left(\left(s_{k} h_{0}+\ldots+s_{k-N} h_{N}\right)^{3}-\gamma\left(s_{k} h_{0}+\ldots+s_{k-N} h_{N}\right)\right)\left[\begin{array}{c}s_{k} \\ \vdots \\ s_{k-N}\end{array}\right]\right\}$

Taking the expectation operator with respect to the source regressor elements $s$ leads to $N+1$ multivariate polynomials in $H$ (the combined channel-equalizer vector) with monomial coefficients given by the second and fourth moments of the source signal. Let these moments be represented by,

$$
\begin{aligned}
\mathcal{R}_{k}^{i} & =\mathrm{E}\left\{s_{m-i} s_{m-j} s_{m-k} s_{m-\ell}\right\} \\
\mathcal{R}_{j}^{i} & =\mathrm{E}\left\{s_{m-i} s_{m-j}\right\}
\end{aligned}
$$

Herein, we assume stationarity so only three (one) lag terms are needed to describe the fourth (second) moments. However, all terms are retained to better show the structure of
(9). The resulting system of equations may be written as,

$$
\left[\begin{array}{c}
\sum_{i} \mathcal{R}_{i}^{i} h_{i}^{3}+3 \sum_{i \neq j} \mathcal{R}_{i}^{i} h_{i}^{2} h_{j}+\sum_{i \neq j \neq k} \mathcal{R}_{j}^{i} h_{i} h_{j} h_{k}-\gamma \sum_{i} \mathcal{R}_{i}^{0} h_{i} \\
\sum_{i} \mathcal{R}_{i}^{i} h_{i}^{3}+3 \sum_{i \neq j} \mathcal{R}_{i}^{i} h_{i}^{2} h_{j}+\sum_{i \neq j \neq k} \mathcal{R}_{j}^{i} h_{i} h_{j} h_{k}-\gamma \sum_{i} \mathcal{R}_{i}^{1} h_{i} \\
\vdots \\
\sum_{i} \mathcal{R}_{i}^{i} h_{i}^{3}+3 \sum_{i \neq j} \mathcal{R}_{i}^{i} h_{i}^{2} h_{j}+\sum_{i \neq j \neq k} \mathcal{R}_{j}^{i} h_{i}^{n} h_{j} h_{k}-\gamma \sum_{i} \mathcal{R}_{i}^{n} h_{i}
\end{array}\right]
$$

Arranging these coefficients into a vector $\overrightarrow{\mathcal{R}}$ we denote (9) as $F(H, \overrightarrow{\mathcal{R}})$. Values of $H$ for which $F(H, \overrightarrow{\mathcal{R}})=\overrightarrow{0}$ define the CMA stationary points.

\section{WHITE SOURCE RESULTS}

Consider the system $F(H, \overrightarrow{\mathcal{R}})$ under the white source condition. In this case, all elements of $\overrightarrow{\mathcal{R}}$ become zero except for $\mathcal{R}_{i}^{i}\left(=\mathcal{R}_{0}^{i} \begin{array}{c}0 \\ 0\end{array}\right), \mathcal{R}_{j}^{i}\left(=\mathcal{R}_{0}^{i}{ }^{2}\right)$, and $\mathcal{R}_{i}^{i}\left(=\mathcal{R}_{0}^{0}\right)$. With the further assumption of equiprobability of each alphabet member (hence $\left.\underset{\mathcal{R}_{0}^{0}}{\mathcal{O}_{0}^{0}} / \mathcal{R}_{0}^{0}=\gamma\right)$, then $(9)$ reduces to

$$
h_{m}\left(\begin{array}{c}
\mathcal{R}_{0}^{0} h_{m}^{2}+3 \mathcal{R}_{0}^{0} \sum_{i \neq m} h_{i}^{2}-\mathcal{R}_{0}^{0} \\
0
\end{array}\right)=0
$$

for $0<m<N$. It is seen that any $h_{m}=0$ trivially solves $f_{m}=0$ (where $f_{m}$ is the $\mathrm{m}^{\text {th }}$ equation of (9). So, only $f_{m}$ for which $h_{m} \neq 0$ need be further considered. Since each nonzero $h_{m}$ in a solution set must solve each equation $f_{m}$, we have that the value of $h_{m}^{2}$ for all non-zero $h_{m}$ in this solution must be the same. Divide the solution set of $F(H, \overrightarrow{\mathcal{R}})=\overrightarrow{0}$ into classes $\mathcal{C}_{K}$, where $K(0 \leq K \leq N+1)$ is the number of non-zero elements of $H$. Then we have each $h_{m} \in \mathcal{C}_{K}$ must solve the equation,

$$
h_{m}=\left\{\begin{array}{l}
0 \\
\pm \sqrt{\frac{\mathcal{R}_{0}^{0}}{0}}
\end{array} \text { for } 1<K<N+1\right.
$$

Notice, that the $K=1$ solution set corresponds to $H$ having only one non-zero entry (of value 1 ) and thus a perfect equalization setting. Such settings are stable since the cost function hessian is the identity matrix (strictly positive eigenvalues). For $K>1$, such the solution sets are saddle points since the cost function hessian may be seen to be strictly nondefinite by a diagonal non-dominance argument. Such results agree with those as in [3], [5]. It is further noted that since every element of a solution set satisfies (11), that there are $3^{N+1}$ stationary points in complex space.

\section{PERFECT EQUALIZATION FOR NON-WHITE SOURCES?}

We may turn the result for white sources around and ask, "for which source correlations do there exist perfect equalization 
parameterizations which are stationary points?". Denote the perfect equalizing parameterizations by $H^{j \star}$ (i.e. the global system parameterization which has all zero entries, except for a 1 (or -1 ) in the $j^{\text {th }}$ position). Evaluating $F\left(H^{j \star}, \overrightarrow{\mathcal{R}}\right)$ yields the conditions

$$
\mathcal{R}_{i}^{i}{ }_{i}^{i}=\gamma \mathcal{R}_{i}^{0} \quad \text { for } 0<i<N
$$

For the iid, uniformly distributed source case, these conditions are trivially satisfied since $\mathcal{R}_{j}^{i}{ }_{j}^{i}=\mathcal{R}_{j}^{i}=0$ whenever $i \neq j$ and $\mathcal{R}_{i}^{i}=\gamma \mathcal{R}_{i}^{i}$ (by definition of $\gamma$ ). However, for the non-white case such a set of conditions is quite restrictive and appears to preclude any possible realistic source correlations. Although it is apparent that several types of "pathological" (in the sense that they bear no information) source sequences would satisfy (12) (e.g. $s(k)=\gamma \forall k$ or $\left.S=\left[\begin{array}{lllll}\gamma & -\gamma & \gamma & -\gamma & \ldots\end{array}\right]\right)$.

\section{SOLVING FOR STATIONARY POINTS}

Direct algebraic solution for the roots of $F(H, \overrightarrow{\mathcal{R}})$ is difficult in the general case, as $F$ is a set of multivariate polynomial equations with many monomials. However, the numerical approach of continuation methods [1], [6] may applied to solve for roots of $F(H, \overrightarrow{\mathcal{R}})$ given a specific $\overrightarrow{\mathcal{R}}$.

In this method, one introduces the homotopy

$$
\Phi(\lambda)=\lambda F+c(1-\lambda) G
$$

consisting of the system of interest (here, $F$ ), a system $G$ with known roots, and a random complex constant $c$. As $\Phi(\lambda)$ is a system with one degree of freedom, the solutions breakup into paths a.e. Now, the desired roots of $F$ may be found by "tracing", in a predictor-corrector fashion, the roots of $\Phi$ from the known roots of $G$ to the roots of $F$ by varying $\lambda$ from 0 to 1.

Mathematically, there are three issues of concern. First, in order to perform such root tracing, we must be guaranteed that there are the same number of roots of systems $F$ and $G$. We construct $G$ such that this is true and follows from Bernstein's Theorem [2] or by recognizing that our system $F$ satisfies the Bezout upper bound. Furthermore, nastiness such as bifurcations or path crossings must be ruled out. This is accomplished herein by the use of the random constant $c$ which puts the systems $F$ and $G$ in relatively general position. A detailed presentation may be found in [6]. Specifically, we choose $G$ to be the CMA-FSE system under uncorrelated source, with the known $3^{N+1}$ solutions as shown above.

\section{PERFORMANCE AND CORRELATION METRICS}

In the equalization problem, an appropriate metric of performance is the level of ISI at a stable stationary channelequalizer parameterization. We introduce a measure of ISI, $\mu_{\text {ISI }}$, to be

$$
\mu_{\mathrm{ISI}}(H)=\frac{\sum_{i=0}^{n}\left|h_{i}\right|-\max _{i}\left|h_{i}\right|}{\max _{i}\left|h_{i}\right|}
$$

As we are motivated to show the effect of source correlation on achieved ISI, we define a metric on non-whiteness as,

$$
\mu_{\mathrm{COR}}(\overrightarrow{\mathcal{R}})=\left\|\overrightarrow{\mathcal{R}}-\overrightarrow{\mathcal{R}}_{w}\right\|
$$

where $\overrightarrow{\mathcal{R}}_{w}$ is the vector $\overrightarrow{\mathcal{R}}$ evaluated for an iid, uniformly distributed source, and $\|\bullet\|$ is the $\ell_{1}$ vector norm.

\section{PERIODIC SOURCES}

Documented failures of CMA-FSE equalizers in actual application in the presence of periodic sources exist [8]. Using continuation methods, the stable stationary points for CMAFSE under periodic sources may be computed. To gain some insight into how the ISI may be effected as the period increases, a monte-carlo computation was performed. For each period $(\rho)$ of length $32,64, \ldots, 8192$, twenty source sequences with equal symbol occurrence were randomly drawn from a 4-PAM, unit variance alphabet, (i.e. $\mathcal{A}=\frac{1}{\sqrt{5}}\{-3,-1,1,3\}$ ). For each sequence, all stable, stationary combined channelequalizer parameterizations corresponding to a 4 tap (halfbaud spaced) channel and 4 tap equalizer when $L=2$ were computed, along with the corresponding $\mu_{\mathrm{COR}}$ and $\mu_{\mathrm{ISI}}$. The results relating $\mu_{\mathrm{ISI}}$ and $\mu_{\mathrm{COR}}$ are plotted in Figure 3 which exhibits a general trend of $\mu_{\text {ISI }}$ with increasing correlation $\left(\mu_{\mathrm{COR}}\right)$. Also plotted is $\mu_{\mathrm{ISI}}$ versus the sequence period $(\rho)$ in Figure 4 . Here as the period increases, $\mu_{\text {ISI }}$ tends to decrease. As $\mathrm{E}\left\{\lim _{\rho \rightarrow \infty} \overrightarrow{\mathcal{R}}=\overrightarrow{\mathcal{R}}_{w}\right\}$, one expects that $\mu_{\text {ISI }} \rightarrow 0$, since $H^{j \star}$ is a stable, stationary point for white sources.

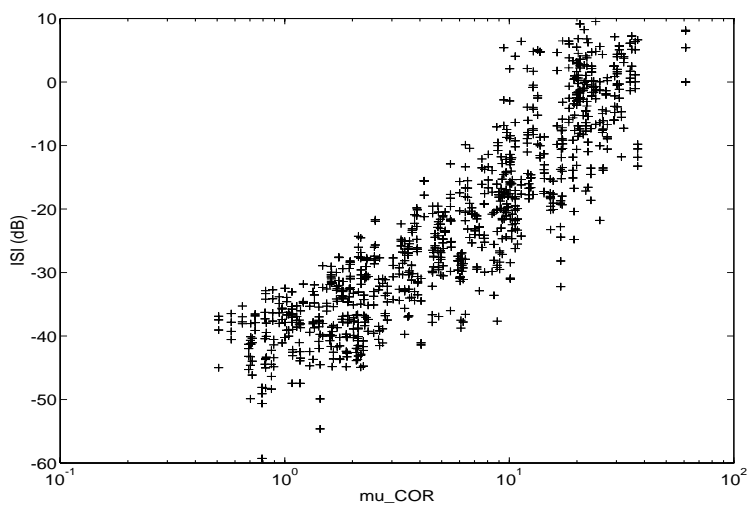

Figure 3: $\mu_{\text {ISI vs } \text { Correlation }}$

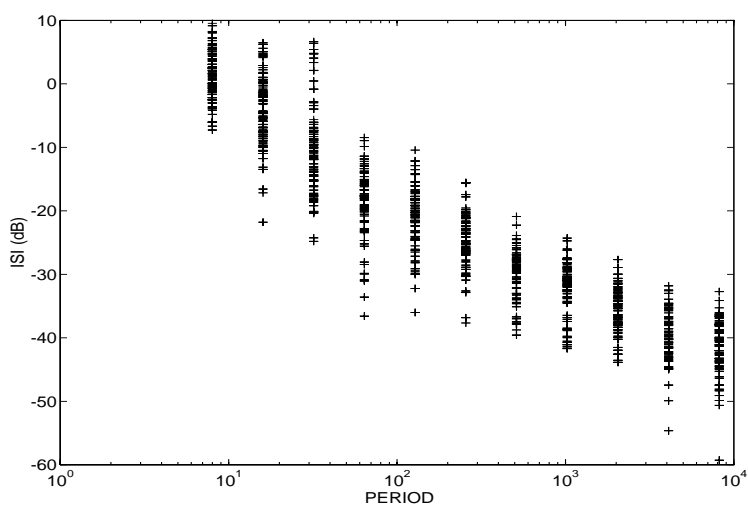

Figure 4: $\mu_{\text {ISI }}$ due to Random Periodic Sources of length $\rho$

\section{MARKOV SOURCES}

As another model of temporally correlated sources, we choose a Markov model. This allows the modelling of a white source as well as temporally correlated sources. Furthermore, since 
convex combinations of Markov transition matrices are themselves valid transition matrices, we can produce a source model which allows smoothly varying $\overrightarrow{\mathcal{R}}$. Then the stationary points can be traced as the source correlation increases. Consider a Markov state transition matrix $\Xi_{w}$ (in which each state represents an alphabet member) which generates a white, uniformly distributed source. (i.e. each matrix element equals $\frac{1}{m}$ for a $m$-ary source). We choose a transition matrix, $\Xi_{c}$ which corresponds to a source correlation of interest and redefine $\Phi$ to be

$$
\Phi(\lambda)=F\left(H, \lambda \Xi_{c}+(1-\lambda) \Xi_{w}\right)
$$

which yields a system wherein performing continuation methods yields a data point of interest at each evaluation of $\lambda$.

An example for the case $\Xi_{c}$ as in Appendix, demonstrates the stationary point movement from $H^{3 \star}$ for a 5 tap system under increasing source correlation. Figure 5 plots the stationary point trajectory as $\lambda$ in (16) is varied from 0 to 1 . The increasing $\mu_{\text {ISI }}$ is plotted as a function of $\mu_{\mathrm{COR}}$ in Figure 6 . The hessian of the cost function was checked at each iteration to verify the stationary point remained a stable one.

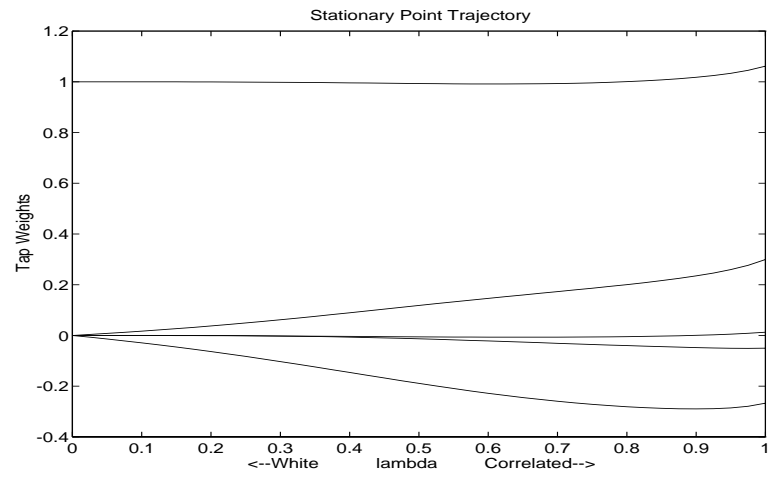

Figure 5: Stationary Points Under Increasingly Non-White Source

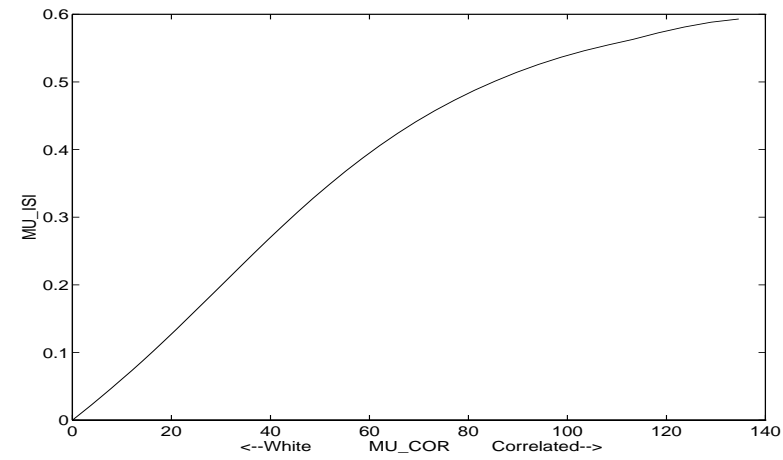

Figure $6: \mu_{\mathrm{ISI}}$ versus $\mu_{\mathrm{COR}}$

\section{CONCLUSION}

In this paper we have presented source correlation conditions which admit perfect equalization under CMA adaptation. Failing such (restrictive) conditions introduces ISI even though a zero ISI equalizer setting exists. Furthermore, the CMA error surface loses its globally convergent property (with equally performing minima) and will now have local minima of differing performance. A method for finding all the stationary points of a system under correlated input is presented. A measure of a correlated source's closeness to admitting perfect equalization $\left(\mu_{\mathrm{COR}}\right)$ has been introduced, as have some examples showing a general trend between this measure and resulting ISI for correlated source models (periodic and Markov).

\section{APPENDIX}

Moments of Markov Source: Define the state vector as $A=\left[\begin{array}{lll}a_{1} & \ldots & a_{m}\end{array}\right]^{\mathrm{t}}$, where $a_{i} \in \mathcal{A}$, and the state transition matrix as $\Xi$, (where the element $\Xi_{i, j}$ is the transition probability from state $i$ to $j$ ), and the steady state probability vector $\Pi=\left[\begin{array}{lll}\pi_{1} & \ldots & \pi_{m}\end{array}\right]^{\mathrm{t}}$ (where $\pi_{i}$ is the steady state probability of state $i$ ), then the temporal correlations for our zero mean process with equiprobable states are then given by (assuming $i \leq j \leq k$ ),

$$
\begin{aligned}
\mathcal{R}_{j}^{i} & =\sum_{\alpha, \beta, \gamma, \delta=0}^{3} a_{\alpha} a_{\beta} a_{\gamma} a_{\delta} \pi_{\alpha}\left(\Xi^{k-j}\right)_{\delta, \gamma}\left(\Xi^{j-i}\right)_{\gamma, \beta}\left(\Xi^{i}\right)_{\beta, \alpha} \\
\mathcal{R}_{i}^{0} & =\sum_{\alpha, \beta=0}^{3} a_{\alpha} a_{\beta} \pi_{\alpha}\left(\Xi^{i}\right)_{\beta, \alpha}
\end{aligned}
$$

Example Transition Matrix:

$$
\Xi_{c}=\left[\begin{array}{cccc}
0 & 0.6 & 0.2 & 0.2 \\
0 & 0 & 0.2 & 0.8 \\
0.8 & 0.2 & 0 & 0 \\
0.2 & 0.2 & 0.6 & 0
\end{array}\right]
$$

\section{REFERENCES}

[1] E. L. Allgower, Numerical Continuation Methods, Springer Verlag, Berlin, 1990

[2] D. N. Bernstein, The Number of Roots of a System of Equations, Functional Analysis and Appl. 9 (1975), 1-4.

[3] I. Fijalkow, F. López de Victoria, and C.R. Johnson Jr., Adaptive Fractionally Spaced Blind Equalization, Proc. DSP workshop, Yosemite 1994.

[4] S. Haykin, Blind Deconvolution, Prentice Hall, Englewood Cliffs, NJ, 1994

[5] Y. Li, Z. Ding, Blind channel identification based on second order cyclostationary statistics, Proc. ICASSP 93, vol. 4, pp. 81-84, 1993.

[6] A. P. Morgan, Solving Polynomial Systems using Continuation for Engineering and Scientific Problems, Prentice-Hall, Englewood Cliff, NJ 1987

[7] L. Tong, G. Xu, T. Kailath, Blind Identification and Equalization Based on Second-Order Statistics: A Time Domain Approach IEEE Trans. Info. Theory, Vol. 40, No. 2, Mar 1994

[8] J. R. Treichler, Observed Misconvergence in the Constant Modulus Adaptive Algorithm, Proc. 25th Asilomar Conference on Signals, Systems and Computers, Nov. 1991 